\title{
EC Projects and Efforts in the Field of Software Quality
}

\author{
Brice Lepape \\ European Commission - DG III / F4 \\ 200 rue de la Loi - B-1049 Bruxelles \\ Tel: 32.2.296 8097 - Fax: 32.2.296 8364 \\ E-mail: ble@dg13.cec.be
}

\begin{abstract}
Software quality has been and still is a continuous concern in the software technology area and numerous projects have contributed to state the art and to improve best practice: results span from contribution to world standards to methods tools and techniques covering various aspects of software quality.

Production of high quality software needs to be tackled from different angles according to the software development process (from user requirements to the product and its evolution) and to the actors involved (users, developers, managers, ...).

The presentation will give a structured overview on how the various issues leading to software quality have been addressed as well as some examples of significant results achieved and on going work in the context of the European RTD programmes.
\end{abstract}

\section{Keywords}

Software Quality - Software Best Practice - ESPRIT

\section{EXTENDED SUMMARY}

Since the beginning of the 80 's the European Commission has launched RTD programmes with the objective to reinforce the technological base and competitiveness of European industry. The flagship programme ESPRIT addresses the Information Technology domain where Software Technology is one of the essential components. In this area more than 300 projects have been launched covering the whole spectrum of 
advanced methods, tools, and techniques to develop and put in use software intensive systems. (CEC Summaries, 1993), (Kuntzmann-Combelles, A., 1989), (KuntzmannCombelles, A., 1993).

ESPRIT has evolved and is still evolving, from its first phase which was mainly technology push to its current status where a market driven approach is taken. During this last decade IT has evolved from a sectoral activity towards a pervasive and generic technology which has become a key asset for the whole economic and social activity. Moreover the convergence of IT and Telecommunications is leading to a profound revolution with the foreseable emergence of the Information Society as described in (CEC White Paper, 1993).

In this fast evolving context Software Technology plays a central role being the means to animate and provide intelligent behaviour of any IT\&C-based systems. The software production demand is broadly outstripping the available resources and the growth of population daily exposed to software based components (often hidden) and applications is accelerating rapidly. It is therefore of paramount importance to develop methods and techniques that enable the software developpers to operate in a more predictable, more user relevant and cost effective manner. Software quality of both product and development process have been a continuous concern since the inception of the programme.

The strategy adopted to address software quality has been pragmatic and evolving, tackling various activities in achieving software quality. From a software product perspective, significant achievements and ongoing work deal with formal methods (eg. LACOS, LOTOSPHRE), user requirements analysis (eg. F3, ORDIT), software metrication and test (eg. MERMAID, MUSIC, COSMOS) and software assessment (eg. SCOPE Bache, R., Bazzana, G. 1994). From a software development perspective, process modelling and improvement (eg. PERFECT, BOOTSTRAP, SQUID), software evolution (eg. MACS, RECYCLE), rapid prototyping (eg. IPTES <Bologna, S., 1993>) and development methods and tools for emerging technologies (e.g. CommonKADS for KBS <Steels, L., Lepape, B., 1992>) have been and still are on the agenda. It is also worthmentioning that the software reuse paradigm (eg. SCALE, REBOOT, EUROWARE), when correctly implemented, will have a significant impact on both software process and products.

Since the beginning of the 90's, due to the rapid evolution of the demand for Software Technology, we are facing a dual dilemma: the current techniques are inadequate to deal with new challenges (e.g. more complexity, more reliability) on one side, and on the other side current practice in software development makes inadequate use of available technologies.

Moreover a smooth transition between new RTD results and their best practice needs to be secured.

The current activities and workprogramme address this dilemma in order to achieve better software quality:

RTD is focusing on the mid-term software quality improvements with a particular emphasis on process modelling and improvement, reuse, user centered developments and safety critical aspects. 
Best Practice (European Software and System Initiative) is dedicated to improving the software development process by means of assessments of current practice, process improvement experiments, training and dissemination.

Trial Applications aim to secure the early adoption of emerging results from RTD by advanced users.

\section{REFERENCES}

CEC White Paper: Growth, Competitiveness, Employment (1993) - Office for Official Publications of the European Union - Luxembourg

CEC Summaries of Projects in Software and Advanced Information Processing (1993) - Office for Official Publication of the European Union - Luxembourg

Kuntzmann-Combelles, A, ESPRIT: Key results of the first phase - IEEE Software, Vol. 6, $\mathrm{N}^{\circ} 6$, (1989)

Kuntzmann-Combelles, A., Luqui, Advancing Europe's Fortunes, IEEE Software, Vol. 6, No6, (1993)

Steels, L., and Lepape, B., Enhancing the knowledge engineering process, Contributions from ESPRIT, North-Holland, Amsterdam, (1992).

Bache, R., and Bazzana, G., Software metrics for product assessment, McGraw-Hill, London, (1994).

Bologna, S., Incremental prototyping technology for embedded real-time systems, Special Issue of Real-time Systems Journal, Vol. 5 N²/3, Kluwer Academic Publishers, (1993). 